\title{
How Cultural Stereotypes and Abuse Affect Family Risk in HIV Transmission
}

\author{
Laurie Nguyen ${ }^{1 *}$
}

\begin{abstract} health policies for HIV.

\section{Keywords}

HIV/AIDS - Social Stigma - LGBTQ

${ }^{1}$ Department of Biological Sciences, University of North Texas

*Faculty Mentor: Dr. Tom Miles
\end{abstract}

Many of the individuals who are affected come from marginalized groups, such as the LGTBQ community, ethnic minorities, and women. Because this disease is so politicized, many of these groups are ostracized, whether it be through discrimination or prejudice. While these individuals do suffer terribly, many social institutions are also affected. This article culls the literature and argues that cultural stereotypes, as well as domestic abuse, are two of the most important determining factors as to how HIV transmission risks will affect the family. Understanding the relationship between cultural stereotypes and abuse can further enhance present research regarding public

\section{Contents}

$\begin{array}{ll} & \text { Introduction } \\ 1 & \text { Why the Family? } \\ 2 & \text { Cultural Stereotypes } \\ 3 & \text { Domestic, Alcohol, and Child Abuse } \\ 4 & \text { Conclusion } \\ & \text { Author Biography } \\ & \text { References }\end{array}$

\section{Introduction}

The Human Immunodeficiency Virus (HIV) is a sexually transmitted infection that slowly degrades the body's immune system, making the individual susceptible to various infections that the body would normally fight against. Many of the individuals who are affected come from marginalized groups, such as the LGTBQ community, ethnic minorities, and women. Because this disease is so politicized, many of these groups are ostracized, whether it be through discrimination or prejudice. While these individuals do suffer terribly, many social institutions are also affected. One of these social institutions is the family.

HIV is a disease that attacks society's perceptions through stigmatization, and often use social ills to perpetuate that stigma. These ills include poverty, domestic abuse, miscommunication of preventative efforts, etc. Generally speaking, these ills are often thought of as negative attributes of an otherwise developed society. When a family member squires this disease, both the individual and the family members are affected. If the family is afflicted with these social ills, they could add to the already troubling psychological burdens that HIV may impose on the family.

Not only this, but the stigma that correlates with the disease are often prevalent in the emotional and mental wellbeing of the family. If the family has no support system outside their unit, this can lead to devastating consequences for both the family and the individual. An example of this can be found in a study done by the National Institute of Mental Health and Neurosciences in India. According to this study, one patient recalls the fear of what would happen should other members of his community found he had HIV. He remembers how much he "wanted to take his wife and daughter away and stay in a remote village so that nobody knew them."[1] Sometimes however, the stigma often associated with HIV can destroy the relationship between an individual and their family. In another study four a patient in Iran who said that when his family found out he had HIV, they had "completely cut their ties with him."[2]

These anecdotes show the complexities HIV can introduce to family life. From fear of discrimination against the community, to rejection from one's loved ones, it's clear that HIV places heavy, emotional wounds on the family. That being said, cultural stereotypes, as well as domestic abuse, are two of the most important determining factors as to how HIV transmission risks will affect the family.

\section{Why the Family?}

To understand the importance of the family in the HIV context, it is imperative to see the family as a major social institution, one that propagates many of society's values onto its members. Despite this, the meaning of the family depends on the cultures 
the families are in. For example, in Western countries, and especially in the United States, the family was thought of as nothing more than a concept.[3] As such, members in the family are considered equals. Whether it be spouses or children, all members are entitled to the same rights as any other individual. In fact, as long as the child demonstrates reasonable maturity, they are even allowed to make their own decisions medically.[4]

Though many groups question the coherence of these bonds, the family has been universally regarded as a moral and cultural foundation for many individuals. Although families consist of separate persons, each person lives together and cares for one another. Hallowachs writes that families are a kind of "kinship, which can only arise within the family and which can be explained only by the family." These bonds create a unique unit within society, since the family has its own interests, knowledge, and morals. This fosters a devotion in each of its members, evidenced by how members are willing to sacrifice their own well-being for other members. In fact, if family members refuse to help each other, they are often stigmatized by the general population, even in Western cultures.

Family members also communicate on a more personal basis than other relationships. This communication often allows the facilitation of information, such as goals, emotions, thoughts, etc. Because of this, the family is oftentimes a vehicle of knowledge that can affect the perceptions of critical events, as well as current attitudes, in society. This is shown over and over again whether it be a mother crying out against the injustice dealt to her children, or relatives mourning the loss of their own, or even members caring for each other in the face of terminal illness; the greater the conflict or injustice, the greater the pain shared between members. [5] These emotions encourage bonding between members. The experiences they have together allowed them to connect with each other and fulfill an emotional need that other relations are incapable of meeting.

Thus, families are, indeed, special institutions that provide "models, examples, and elements of teaching...they express the general attitude of the group."'[6] In other words, the family is a gateway that provides a guideline for how someone should act. Families who live in communities have an idea of societal norms and would know whether their members were behaving deviantly.

As such, within the context of HIV, misinformation can spread like wildfire. Stigma, prejudice, and discrimination can be propagated through the family, and if it's surrounded by a community that holds such beliefs, that information can be even more devastating. This can also be said for when its family members are suffering from domestic abuse. Abuse perpetuates fear, as well as helplessness, that can often leave family members susceptible to various STIs, HIV included. Because of this, it's important for health professionals and mental health professionals to know how these factors affect the family, as well as their risks in attaining HIV.

\section{Cultural Stereotypes}

Society comprises a vast amount of values, cultures, and languages that describe some, if not most, human values and behavior. Communities have held their own unique perspectives and ideals, much of which allows people to develop a variety of relations with one another. Depending on that society, many families are often influenced by both positive and negative connotations of that society, especially with a politicized disease such as HIV. There are universal aspects in each society that play a role in determining just how much of a risk there is in believing those aspects, especially negative ones. And among these dark variables are cultural stereotypes.

Cultures tend to have different stereotypes when it comes to classifying people who are perceived as different. These stereotypes have been used to perpetuate many ideas that are otherwise insulting to others. For instance, African Americans tend to believe in masculinity, and that heterosexual relationships are the norm. To be homosexual is considered abnormal. Because HIV is often thought of as a gay man's disease, African Americans may be reluctant to receive help, especially when they are first afflicted with HIV. In fact for many African Americans, "negative attitudes toward HIV prevention are linked to racism."[7] The same stigma is similar to that found in Asian communities. In Asian cultures where the family structure is considered essential, patriarchal heads typically lead these families. Like African American communities, being "gay" is often thought of as a devastating identity that can bring about the downfall of the family. If these stereotypes spread these families, these conflicts "between ethnic identity and sexual identity might hinder safer sex behaviors."[8] In fact, in Asian communities, prevailing beliefs denote homosexual activity as an evil phenomenon that arose from the West. Because of this, many individuals may not be comfortable with their behavior. After all, family honor comes before an individual, and as such, they may continue to hold up their family's reputation, but in exchange, continue their behavior secretly. This could lead to hesitation for many people to seek out HIV prevention measures or treatments.

Racism has also played a part in contributing to these cultural stereotypes. From the age-old illustration of the conniving Asian, to the lazy Black man, there's no doubt that racism has impacted many families. For example, while many advertise that the rates of infection of HIV have gone down, these rates only apply to Caucasians. Because of the added stigma of being homosexual, alongside being a minority, there is a risk that an individual could lose the support of their family. For instance, oftentimes, to gain the support of their families, African Americans would more likely identify with being "black", rather than gay.[9] What's more, perceived racism can also play a factor into determining whether the individual goes to a health professional at all. Surprisingly enough, the higher the perceived racism, the higher the chances that an individual would go to get tested for HIV.[10] As such, it may not be that detrimental. However, in another study done in France, researchers have found that discrimination, by race, 
HIV status, or gender, is quite prevalent in HIV positive individuals. Below is a figure of the HIV rates as a result of these prevailing gender views:

This data set, taken from the Center for Disease Control and Prevention, (2017) shows that African American homosexual contact is among the highest of these statistics. This could be due to the shame that these individuals may have, which leads them to refuse treatment and prevention regarding HIV. The same can be said for Latino/Hispanic individuals. And while this data does, in fact, show that homosexuals still have most of the infection rates, heterosexual contact in HIV transmissions are beginning to rise as well.

Yet another example of these stereotypes are gender roles. In the past HIV was often seen as a disease that only affected gay communities and were treated as such. Now however, HIV has slowly shifted to affect women. This is often because many cultures place women in household roles and be subservient to men. This has caused many women to be financially insecure, and to have to rely on their husbands. What's more, because many women are often required to stay home, they have gaps in their education that could allow them to learn more about HIV. These gender roles however, aren't only limited to women. Gay men were also subjected to these cruelties. Oftentimes, In Hispanic culture, the term "machismo" is defined as a man who emulates traditionally masculine characteristics, such as having courage, strength, and dominance. In the context of sex, this man "penetrates" their partners, man or women. In other words, as long as the man is on told during sex, then they do not qualify as being "gay." Again, because of this stereotype, this offers many opportunities for HIV transmission. Any man who isn't dominant will be classified as gay, and as such, as expected to act more feminine. Upholding masculine pride in the family is considered important; having multiple sex partners, caring for wives, and providing financial support is a prevalent theme.

Religion has also been used to introduce these stereotypes within families. Religion has been shown to have a positive effect in curbing HIV transmissions, such as promoting abstinence, community, providing support, and assisting in mental health. For those infected by HIV, religion can also offer guidance. But despite these attributes, religion can also have a negative effect on the psychosocial factors of the disease. Homophobic attitudes, especially in conservative Christian groups, have tended to classify HIV/AIDs as a "gay man's disease, as was seen with Vice President Mike Pence, a Christian politician who has been known to pass legislation against the LGBT community. Due to Pence's slow position on the needle-exchange program, he had indirectly allowed the HIV outbreak to spread. Many religious authorities have also perpetuated the HIV/AIDs stereotype, in which HIV was seen as a punishment from God. While there are groups that advocate about using religion to support those with HIV, there are also those who believe they could never get the disease, simply because they play the role of the "good Christian"; they go to church, try not to sin, and follow other church rules. Religious has also been used to promote "traditional family values", and more often than not, shames those who aren't heterosexual. These stereotypes can linger within many churches and can have devastating consequences should a family member deviate.

Cultural stereotypes have oftentimes been used to label individuals, with many negative connotations attached. From ethnic to gender stereotypes, to even social institutions such as religion, these harmful ideas hide factors that contribute to HIV, psychological issues that might've been dealt with in a more professional manner.

\section{Domestic, Alcohol, and Child Abuse}

Domestic abuse comes in many forms. From emotional, to financial, to physical, domestic abuse has long been a sinister culprit in perpetuating the risk of HIV transmission. Not only does it make the partner uncomfortable for even negotiating for safer sex, but also puts them at risk for HIV and other STIs and can open the door to other comorbid disorders that could affect an individual to the point where they place themselves in potentially dangerous situations, situations where they can attain HIV easily. Domestic abuse, most importantly, can damage the structure of the family, and foster miscommunication that allows HIV infection to become more prevalent.

Defined by The National Domestic Violence Hotline, domestic violence, also known as intimate partner violence, is a series of behaviors used by one partner to dominate the other. This can include controlling finances, emotions, and isolating the other. One of the ways domestic violence has played a part in transmitting the disease is by promoting harmful gender roles.[11] As stated before, across many cultures, men were more likely to have powerful roles than women. This can facilitate this illustration that women are the weaker sex, and as such, must rely on men for even the basic of necessities. For instance, in South Africa, it is believed that men are more powerful and stronger than women, and so, men should be in charge of a relationship between the two.[12] Because of this, South Africa has power imbalances between the two genders that can raise a woman's risk for sexually transmitted infections.[13] With this assumption, a woman is more likely to submit to a man, especially if they are dependent on a man for a variety of necessities such as food, shelter, ect. Thus, it can be difficult for women to negotiate having safer sex without the fear of being abandoned, abused, or even abused.

What often accompanies domestic abuse is the potential for comorbid disorders. One such example of this is alcohol abuse. Typically speaking, families who have higher rates of HIV infection tend to have higher rates of partner violence and alcohol abuse.[14] A study done in Rwanda found that alcohol abuse impedes relationships between family members, and thus, can increase the risk rate for HIV. A counselor in the study had even reported that a child was "very concerned by her family conflicts where the father was not caring toward the family and beats the mother a lot when he gets drunk." Accompanying this abuse, when an individual is impaired, 


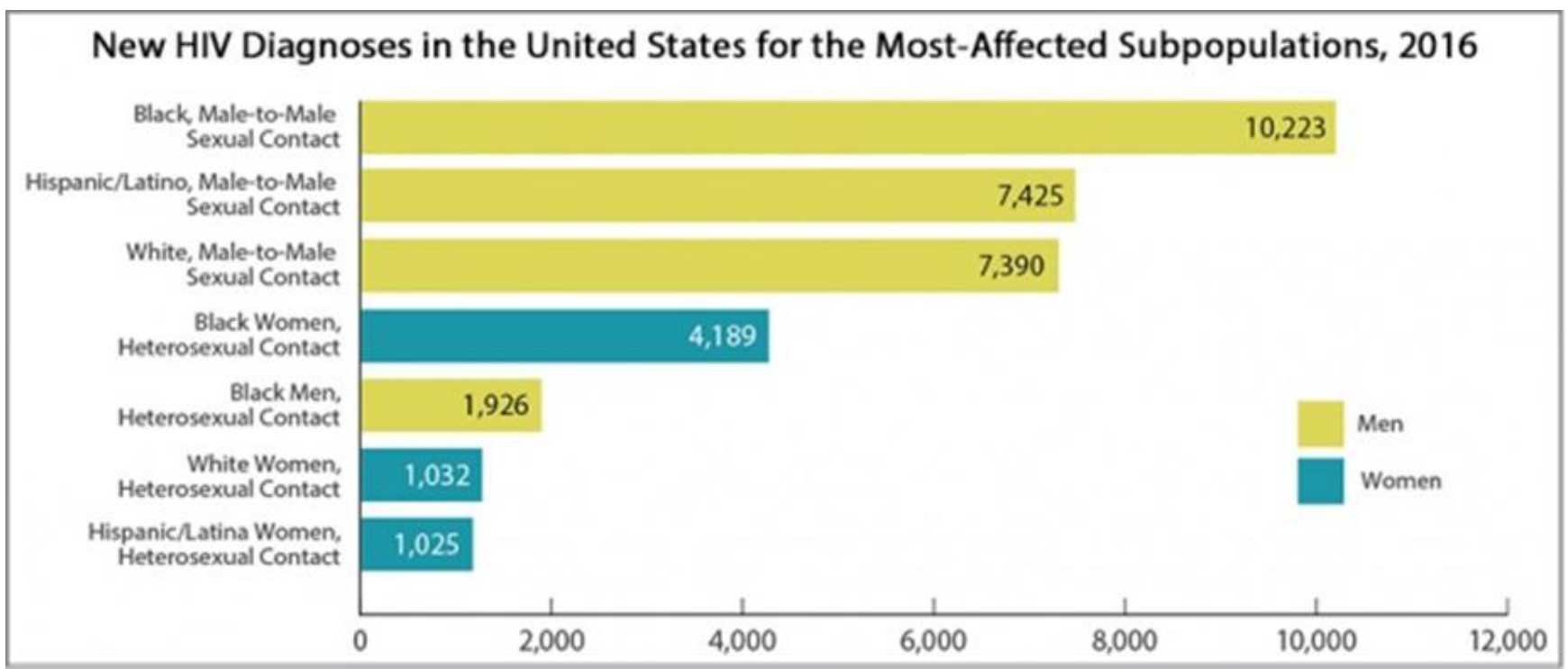

Figure 1. HIV Diagnoses by Transmission Category

they may do something that they may not otherwise consider. For instance, when under the influence, the individual may impose themselves on their partner. When the partner is too frightened to do anything, their risk of being infected with any sexually transmitted diseases increases. One study had shown that "maternal alcohol abuse was significantly associated with paternal alcohol abuse." In a similar study, drug abuse by either parent has led to a strong correlation in many childhood traumas.[15] It's a cycle of suffering that many individuals have gone through. However, this cycle has devastating consequences concerning HIV. Because of how alcohol impairs judgement, this can promote risky behaviors that can put an individual at risk for attaining HIV. Even after an individual is infected with HIV, they may continue to indulge in detrimental alcoholic behaviors. According to a study done in Vietnam, individuals who indulge in high alcohol consumption is generally correlated with non-treatment adherence for those living with HIV [16], alongside downgrading an individual's immune system to the point where they become vulnerable to other diseases. Because the family is responsible for an individual's care, there's concern as to whether or not the individual has the same support as others.

This leads to the topic of childhood abuse. According to RAINN, an organization dedicated to fighting and spreading awareness of child abuse, child abuse is defined as harm done to a minor, either physically, psychologically, sexually, or emotional. Child abuse is outed by society, though oftentimes the victims don't report the abuse. This could be due to helplessness, cultural stereotypes, and gender roles that, for the most part reinforce the silence of the victims. Even so, there's no doubt that child abuse does hurt the family; when boundaries are violated, and the trust between a trusted caretaker and child is broken, this can lead to many consequences. In a study done in Egypt, children who lived in conditions where they have a greater risks of being abused tended to come from families who lived in poverty, have parents who had a history of being abused, and lived in household where domestic violence was normal.[17] Children who were also sexually abused had long-term health risks, including an increased risk of acquiring HIV. In a research study published in the American Journal of Public Health, victims of child sexual abuse were four times more likely to have "worked as a prostitute during their lifetime." They were also to have reported to have engaged in unprotected sex. Child sexual abuse has had a devastating impact on HIV risk rates. Along with domestic abuse, child sexual abuse differs in other forms of child abuse in that the child is used for sexual gratification. Because HIV is a blood-borne disease, and can be transmitted through sexual contact, children who have suffered from sexual assault may be at a greater risk for HIV.

Domestic abuse not only forces the victim to subject to the abuser, leading them to not be able to negotiate for protecting against diseases like HIV. What's more, domestic abuse is connected to other forms of abuse, such as substance abuse, and even childhood trauma. The psychological detriments associated with these conditions negatively impact both family life and HIV transmission rates.

\section{Conclusion}

Despite the fact health professionals know the biological basis of HIV/AIDs, many people are only just beginning to understand the societal aspects associated with it. Cultural stereotypes and traditional gender roles can be used to promote an environment of violence. This, in turn, creates isolation, fear, and guilt, which can play a role in creating an inhospitable situation for both the caretakers and the child. What's more, domestic abuse and other forms of abuse can introduce other psychological burdens that can further propagate the risk of HIV transmissions. From the inability to negotiate for safer 
sex, to the risk of substance abuse, to even learned helplessness, these types of abuse add to the risk of contracting HIV.

Even so, the family is resilient. It is made up of individuals who share similar experiences, have similar beliefs, and have a unique relationship with one another that many can't find elsewhere. The family is also living in the context of the environment surrounding them; culture, language, and even small prejudices often affect the family, especially in strengthening or weakening their ties with each other. Understanding the relationship between cultural stereotypes and abuse can further enhance present research regarding public health policies for HIV.

\section{Author Biography}

Laurie Nguyen received her Bachelor's degree from UNT in 2018. She is currently applying to graduate programs in counselling psychology.

\section{References}

[1] VAS Krishna, Ranbir S Bhatti, Prabha S Chandra, and Srilatha Juvva. Unheard voices: experiences of families living with HIV/AIDS in India. Contemporary Family Therapy, 27(4):483-506, 2005.

https://doi.org/10.1007/s10591-005-8235-9

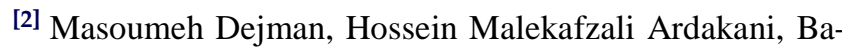
hareh Malekafzali, Ghobad Moradi, Mohammad Mehdi Gouya, Zahra Jorjoran Shushtari, Seyed Ahmad Seyed Alinaghi, and Minoo Mohraz. Psychological, social, and familial problems of people living with HIV/AIDS in Iran: A qualitative study. International journal of preventive medicine, 6, 2015.

https://dx.doi.org/10.4103\%2F2008-7802.172540

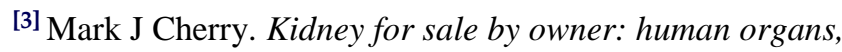
transplantation, and the market. Georgetown University Press, 2015.

[4] Joanne Whitty-Rogers, Marion Alex, Cathy MacDonald, Donna Pierrynowski Gallant, and Wendy Austin. Working with children in end-of-life decision making. Nursing Ethics, 16(6):743-758, 2009.

https://doi.org/10.1177\%2F0969733009341910

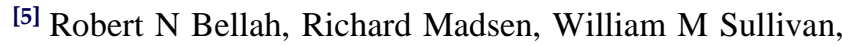
Ann Swidler, and Steven M Tipton. Habits of the heart: Individualism and commitment in American life. Univ of California Press, 2007.

[6] Maurice Halbwachs. On collective memory. University of Chicago Press, 1992.

[7] Chandra L Ford, Mark Daniel, Jo Anne L Earp, Jay S Kaufman, Carol E Golin, and William C Miller. Perceived everyday racism, residential segregation, and HIV testing among patients at a sexually transmitted disease clinic. American journal of public health, 99(S1):S137S143, 2009.

https://ajph.aphapublications.org/doi/abs/10.2105/AJPH.2007.120865

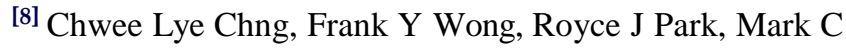
Edberg, and David S Lai. A model for understand- ing sexual health among Asian American/Pacific Islander men who have sex with men (MSM) in the United States. AIDS Education and Prevention, 15(1 Supplement):21-38, 2003.

https://doi.org/10.1521/aeap.15.1.5.21.23611

[9] Larry M Gant, Patricia A Stewart, and Vincent J Lynch. Social workers speak out on the HIV/AIDS crisis: Voices from and to African American communities. Greenwood Publishing Group, 1998.

[10] Elise Marsicano, Rosemary Dray-Spira, France Lert, Cindy Aubrière, Bruno Spire, Christine Hamelin, and ANRS-Vespa2 Study Group. Multiple discriminations experienced by people living with HIV in France: results from the anrs-vespa2 study. AIDS care, 26(sup1):S97S106, 2014.

https://doi.org/10.1080/09540121.2014.907385

[11] Seth C Kalichman, Leickness C Simbayi, Allanise Cloete, Chauncey Cherry, Anna Strebel, Moira O Kalichman, Tammy Shefer, Mary Crawford, Mokgethi Thabalala, Numvo Henda, et al. HIV/AIDS risk reduction and domestic violence prevention intervention for south african men. International Journal of Men's Health, 7(3), 2008.

DOI: 10.31419/jmh.0703.255

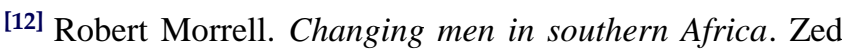
books, 2001.

[13] Paul Farmer, Margaret Connors, and Janie Simmons. Women. Poverty \& AIDS: Sex, Drugs and Structural Violence Series in Health and Social Justice, 1996.

[14] Sumona Chaudhury, Felicity L Brown, Catherine M Kirk, Sylvere Mukunzi, Beatha Nyirandagijimana, Josee Mukandanga, Christian Ukundineza, Kalisa Godfrey, Lauren $\mathrm{C} \mathrm{Ng}$, Robert T Brennan, et al. Exploring the potential of a family-based prevention intervention to reduce alcohol use and violence within HIV-affected families in Rwanda. AIDS care, 28(sup2):118-129, 2016.

https://doi.org/10.1080/09540121.2016.1176686

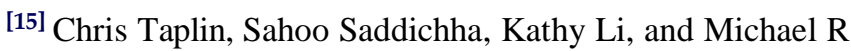
Krausz. Family history of alcohol and drug abuse, childhood trauma, and age of first drug injection. Substance use \& misuse, 49(10):1311-1316, 2014.

https://doi.org/10.3109/10826084.2014.901383

[16] Bach Xuan Tran, Long Hoang Nguyen, Cuong Tat Nguyen, Huong Thu Thi Phan, and Carl A Latkin. Alcohol abuse increases the risk of HIV infection and diminishes health status of clients attending HIV testing services in Vietnam. Harm reduction journal, 13(1):6, 2016.

https://doi.org/10.1186/s12954-016-0096-z

[17] Mohammed N Al Dosari, Mazen Ferwana, Imad Abdulmajeed, Khaled K Aldossari, and Jamaan M Al-Zahrani. Parents' perceptions about child abuse and their impact on physical and emotional child abuse: A study from primary health care centers in Riyadh, Saudi Arabia. Journal of family \& community medicine, 24(2):79, 2017. 